\title{
Rapid detection of cryptococcal antigen by a flow assay
}

\author{
Graziano Bargiggia, Silvia Corbellini, Gaia Ortalli, Fausto Moioli, Claudio Farina \\ Microbiology and Virology Laboratory, Papa Giovanni XXIII Hospital, Bergamo, Italy
}

\begin{abstract}
Summary
Cryptococcosis is a life-threatening infection caused by Cryptococcus neoformans and C. gattii. Tests for quick detection of the cryptococcal antigen are needed. This study compares the performance of a lateral flow assay (LFA) to the latex agglutination method. Thirty-five cryopreserved positive samples (sera and cerebrospinal fluids) plus three negative sera for control have been examined. LFA does not need high-temperature incubation or enzyme pre-treatment. All the results, except for one serum, agree with previous obtained with latex agglutination method. LFA has an important clinical utility for its rapidity and sensitivity, and it also can be used as a point-of-care test.
\end{abstract}

\section{Introduction}

Typically, cryptococcosis is diagnosed by testing cerebrospinal fluid (CSF) by culture, India ink microscopy, or cryptococcal antigen (CrAg) $(1,2)$. CrAg can be detected by enzyme immunoassays (EIA) methods or by agglutination of sensitized latex particles (LA). However, these methods require a preliminary 15-min centrifugation; for EIA assay is also mandatory a 45min procedure (sample incubation, enzyme conjugation, substrate

Correspondence: Claudio Farina, UOC Microbiologia e Virologia, ASST Papa Giovanni XXIII, Piazza OMS 1, 24127 Bergamo, Italy.

Tel: +39.035.2673666 - Fax: +39.035.2674921.

E-mail: cfarina@asst-pg23.it

Key words: Cryptococcosis, antigen, LFA.

Contributions: GB and GA performed the tests; FM and CF participated in the study design and in the evaluation of results; SC drafted the article

Conflict of interest: the authors declare no potential conflict of interest.

Received for publication: 4 August 2017.

Revision received: 5 September 2017.

Accepted for publication: 5 September 2017.

CCopyright G. Bargiggia et al., 2017

Licensee PAGEPress, Italy

Microbiologia Medica 2017; 32:6977

doi:10.4081/mm.2017.6977

This article is distributed under the terms of the Creative Commons Attribution Noncommercial License (by-nc 4.0) which permits any noncommercial use, distribution, and reproduction in any medium, provided the original author(s) and source are credited. and stop solution), while LA necessitates an incubation step with pronase enzyme (1). Moreover, the test may be difficult to read, notably in case of weak agglutination (8). For these reasons, FDA approved the CrAg Lateral Flow Assay IMMY CrAg®LFA (Imprimis Pharmaceuticals Inc., Norman, OK, USA), an immunochromatographic dipstick assay for the detection of $\mathrm{CrAg}$ capsular polysaccharide.

In this study, the performance of the LFA for CrAg detection was evaluated at Papa Giovanni XXIII Hospital, Bergamo, Italy.

\section{Materials and Methods}

Thirty-eight (33 sera and 5 CFS) consecutively collected clinical specimens were tested with CrAg Lateral Flow Assay IMMY CrAg ${ }^{\circledR}$ LFA (Imprimis Pharmaceuticals Inc. San Diego, CA, USA), in comparison with CALAS ${ }^{\mathrm{TM}}$ agglutination kit ( $\mathrm{CrAg}$ Latex Agglutination System, Meridian Bioscience Inc., Cincinnati, OH, USA). Thirty-five (30 sera and 5 CSF) were collected from 24 HIV-positive patients ( $83 \%$ males, 17\% females; mean age: 40 , range: $26-68$; admission to Gastroenterology 5\%, General Medicine 5\%, Infectious Diseases 90\%), with a cultureproven diagnosis of cryptococcosis. Three additional tested samples were negative. All specimens were preserved at $-80^{\circ} \mathrm{C}$ before experimental testing.

LFA is an immunocromatographic test dipstick that implies gold-conjugated monoclonal antibodies bounded. If CrAg is present in a sample, antibodies bind the antigen. The gold-antibodyCrAg complex migrates by capillarity up the strip, combined with the immobilized monoclonal antibodies against $\mathrm{CrAg}$ and forms red line. The test is performed by adding one drop of diluent to 40 $\mu l$ of patient specimens. The dipstick is inserted, incubated at room temperature for 10 minutes and then read.

A standard curve based on the increasing intensity of the positive bands, giving a numeric value (0-4) has been proposed. Titres measured with CALAS ${ }^{\mathrm{TM}}$ method were visually compared, after a 10-minute incubation, with the intensity of the lines of LFA test, to try to obtain a semi-quantitative assessment. First of all, a negative sample and four strips with positive bands of increasing intensity were used to construct a standard curve. To each of these bands a numeric value ranging from 1 to 4 was assigned (Figure 1).

\section{Results}

Qualitative LFA results on CSF samples were concordant with those obtained with LA (sensitivity 100\%). Among sera, there was one case of discrepancy between the two methods: positive with LA but negative with LFA (sensitivity $96.6 \%$ ) because of a very low antigen titre (1:4). The three negative controls showed no 
lines in LFA (specificity 100\%). Titres measured with CALAS ${ }^{\mathrm{TM}}$ method are shown in Table 1. LA titre comparison with LFA test confirmed that low levels of intensity of the bands of the LFA test corresponded to a low titre measured with CALAS ${ }^{\mathrm{TM}}$, while at higher intensity bands corresponded higher titres. However, scores ranging from 2 to 3 present overlap of titres ranging from 1:1024 to $1: 8192$.

For two patients affected by AIDS, more samples collected at different intervals of time were tested to verify the presence of CrAg over time (Table 2). Moving away from primary infection, a reduction both in titre measured by the LA test and in intensity of the bands by LFA test was observed.

\section{Discussion and Conclusions}

Cryptococcosis is a life-threatening infection caused by Cryptococcus neoformans and C. gattii (4-6). Current diagnostic procedures of cryptococcosis, whether direct or indirect, have limitations. They require both a particular expertise to avoid false positive and false negative results (Indian ink staining) or a prolonged preparation time (centrifugation and/or pronase incubation) to reach adequate sensitivity (1). This latter limitation is overcome with the CrAg Lateral Flow Assay IMMY CrAg ${ }^{\circledR}$ LFA, that is a rapid and easy to use assay. It can be directly performed on the native sample (serum, plasma, whole blood, urine and FSC) and results can be obtained in less than 15 minutes (it has shelf stability at room temperature, and it does not need heat or enzyme treatment), and requires only a minimum training (7). A semi-quantitative use of the test is possible with serial dilutions of the sample (2) and quantification using laser thermal contrast has been reported (1).

LFA specificity was calculated in previous studies to be between 99.33 and 100\% (5). In a large study, Hansen et al. found an excellent concordance with the Meridian EIA test but without complete access to patient-level data, they were unable to fully conclude on the origin of discrepancies (3). We found specificity at $100 \%$ on CSF tested. Instead, one serum was considered false negative (specificity $100 \%$ and sensitivity $96.6 \%$ ). Titres measured with CALAS $^{\mathrm{TM}}$ method were compared with the intensity of the lines of LFA test, to try to obtain a semi-quantitative assessment. We observed that at low levels of intensity of the bands of the LFA test corresponded to a low titre measured with CALAS ${ }^{\mathrm{TM}}$, while at higher intensity bands corresponded more high titres.

In conclusion, LFA is ease of use (practicality and reading),

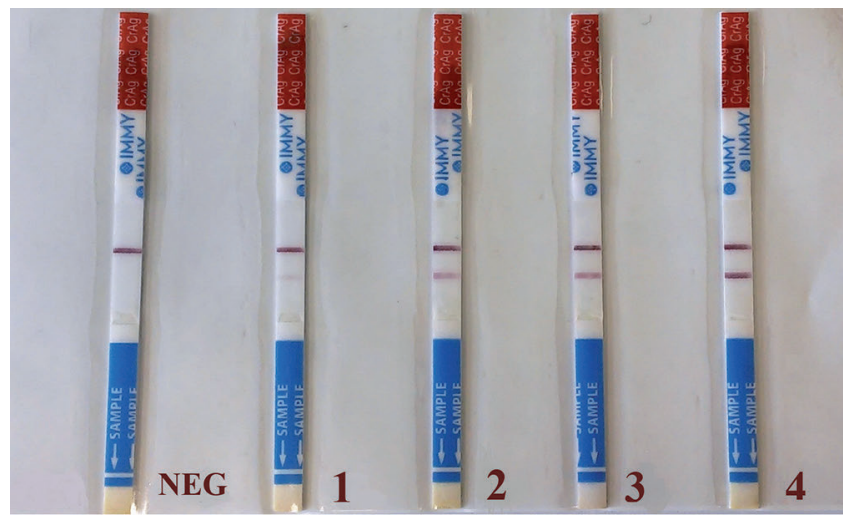

Figure 1. Score of intensity lines.

Table 1. Titres ordered per material.

\begin{tabular}{lcl} 
Titre & Serum & CFS \\
$1: 131072$ & 1 & 0 \\
$1: 32768$ & 1 & 0 \\
\hline$>1: 20480$ & 1 & 0 \\
$1: 16384$ & 1 & 0 \\
\hline $1: 8192$ & 4 & 1 \\
$1: 4096$ & 4 & 0 \\
\hline $1: 2048$ & 3 & 0 \\
$1: 1024$ & 1 & 0 \\
\hline $1: 512$ & 2 & 0 \\
$1: 256$ & 3 & 1 \\
\hline $1: 128$ & 1 & 0 \\
$1: 64$ & 2 & 1 \\
\hline $1: 32$ & 1 & 0 \\
$1: 16$ & 3 & 1 \\
\hline $1: 8$ & 1 & 1 \\
$1: 4$ & 1 & 0 \\
\hline $1: 2$ & 0 & 0 \\
Total & 30 & 5 \\
\hline
\end{tabular}

Table 2. Follow up of two patients.

\begin{tabular}{|c|c|c|c|c|c|}
\hline Sample & Titres: dilution & Standard LFA & Type of sample & Year & Ward \\
\hline \multicolumn{6}{|c|}{ Patient 1} \\
\hline 1 & 1:4096 & 3 & Serum & 2001 & Infectious diseases \\
\hline 2 & $1: 4096$ & 2 & Serum & 2001 & Infectious diseases \\
\hline 3 & $1: 64$ & 1 & Serum & 2003 & Infectious diseases \\
\hline 4 & $1: 16$ & 1 & Serum & 2005 & Infectious diseases \\
\hline 5 & $1: 4$ & NEG & Serum & 2014 & Infectious diseases \\
\hline \multicolumn{6}{|c|}{ Patient 2} \\
\hline 1 & $1: 8192$ & 2 & Serum & 2001 & Infectious diseases \\
\hline 2 & $1: 4096$ & 2 & Liquor & 2001 & Infectious diseases \\
\hline 3 & $1: 8$ & 1 & Liquor & 2001 & Infectious diseases \\
\hline
\end{tabular}


quick (results available in less than 15 minutes) and fully appropriate for diagnosis of cryptococcosis. A semi-quantitative use of the test can be recommended to detect positivity for cryptococcal antigen as a screening test, requiring further evaluation with LA to define the final titration.

\section{References}

1. Boulware DR, Rolfes MA, Butler EK, et al. Multisite validation of cryptococcal antigen lateral flow assay and quantification by laser thermal contrast. Emerg Infect Dis 2014;20:45-53.

2. Brizendine KD, Baddley JW, Pappas PG. Predictors of mortality and differences in clinical features among patients with Cryptococcosis according to immune status. PLoS One 2013;8 e60431.

3. Hansen J, Slechta ES, Gates-Hollingsworth MA, et al. Large- scale evaluation of the immuno-mycologics lateral flow and enzyme-linked immunoassays for detection of cryptococcal antigen in serum and cerebrospinal. M fluid. Clin Vaccine Immunol 2013;20:52e5.

4. Harris JR, Lockhart SR, Debess E, et al. Cryptococcus gattii in the United States: clinical aspects of infection with an emerging pathogen. Clin Infect Dis 2011;53:1188-95.

5. Jenney A, Pandithage K, Fisher DA, et al. Cryptococcus infection in tropical Australia. J Clin Microbiol 2004;42:3865-8.

6. Lortholary O, Poizat G, Zeller V, et al. Longterm outcome of AIDS-associated cryptococcosis in the era of combination antiretroviral therapy. AIDS 2006;20:2183-91.

7. McMullan BJ, Halliday C, Sorrell TC, et al. Clinical utility of the cryptococcal antigen lateral flow assay in a diagnostic mycology laboratory. PLoS One 2012;7:e49541.

8. Tanner DC, Weinstein MP, Fedorciw B, et al. Comparison of commercial kits for detection of cryptococcal antigen. J Clin Microbiol 1994;32 1680-4. 